\title{
Major Component Diseases, Autoantibodies, and Typical Clinical and Endocrinological Findings Observed in Each Type of Polyglandular Autoimmune Syndrome
}

\author{
Hidekatsu Yanai
}

In this issue (P159), Oleo et al report a patient with polyglandular autoimmune syndrome (PAS) 3B [1], and they concluded that it is important to monitor for the development of new autoimmune diseases in patients with PAS. I completely agree with their conclusion.

To detect the newly onset of autoimmune diseases, we should have sufficient knowledge about PAS. We previously reported clinical, endocrinological and immunological characteristics of Japanese patients with PAS3A [2]. The relationship of immunological phenotype with clinical and endocrinological phenotypes is very complicated. In type 1 diabetic patients with PAS3A, approximately $80 \%$ of patients showed the positivity for anti-glutamic acid decarboxylase antibody; however, intrinsic insulin secretion has been preserved in almost half of PAS3A patients. Furthermore, although the positivity for anti-thyroid peroxidase antibody was observed in about $70 \%$ of PAS3A patients, only $15 \%$ of PAS3A patients showed hypothyroidism or thyroid swelling. To predict the development of new autoimmune disease, we should be familiar with autoantibodies and clinical manifestation of each type of PAS.

Major component diseases, autoantibodies, and typical clinical and endocrinological findings observed in each type of PAS which was made by modification of previous reports are shown in Table 1 [3-5]. In addition to the information in Table 1, I would like to mention that the measurement of autoantibody to interferon- $\omega$ is useful to detect PAS type $1[6,7]$.

I really hope that this editorial will help physicians and scientists to elucidate the pathophysiology and treatment for PAS.

\section{Competing Interests}

The author declares that he has no competing interests concerning this article.

Manuscript submitted September 22, 2017, accepted October 4, 2017

Department of Internal Medicine, National Center for Global Health and Medicine, Kohnodai Hospital, 1-7-1 Kohnodai, Ichikawa, Chiba 272-8516, Japan. Email: dyanai@hospk.ncgm.go.jp

doi: https://doi.org/10.14740/jem452w

\section{References}

1. Oleo IERD, Saldarriaga MM, Silverman M, Thibaud S, Sponholz LR. Polyglandular autoimmune syndrome Type 3B: hyperthyroidism and pernicious anemia a reminder of the past. J Endocrinol Metab. 2017;7(5):159-161.

2. Moriyama S, Yoshikawa R, Katsuyama H, Hamasaki H, Adachi H, Yanai H. Clinical, endocrinological and immunological characteristics of Japanese patients with autoimmune polyglandular syndrome type 3a. J Endocrinol Metab. 2016;6(2):46-51.

3. Betterle C, Dal Pra C, Mantero F, Zanchetta R. Autoimmune adrenal insufficiency and autoimmune polyendocrine syndromes: autoantibodies, autoantigens, and their applicability in diagnosis and disease prediction. Endocr Rev. 2002;23(3):327-364.

4. Hamasaki H, Yanai H, Hiraishi C, Moriyama S. A significant discrepancy between endocrinological and clinical phenotype and immunological phenotype in autoimmune polyglandular syndrome type 3. J Med Cases. 2012;3(1):1-3.

5. Takazono T, Izumikawa K, Nagayoshi Y, Tanaka A, Mihara T, Kosai K, Saijo T, et al. Evaluation of the Cica Fungi Test Candida, a novel serum Candida mannan antigen kit, and its comparison with Cand-Tec in candidemia patients. Jpn J Infect Dis. 2011;64(2):116-120.

6. Larosa MDP, Mackenzie R, Burne P, Garelli S, Barollo S, Masiero S, Rubin B, et al. Assessment of autoantibodies to interferon-omega in patients with autoimmune polyendocrine syndrome type 1: using a new immunoprecipitation assay. Clin Chem Lab Med. 2017;55(7):1003-1012.

7. Bruserud O, Oftedal BE, Landegren N, Erichsen MM, Bratland E, Lima K, Jorgensen AP, et al. A Longitudinal Follow-up of Autoimmune Polyendocrine Syndrome Type 1. J Clin Endocrinol Metab. 2016;101(8):29752983. 
Table 1. Major Component Diseases, Autoantibodies, and Typical Clinical and Endocrinological Findings Observed in Each Type of Polyglandular Autoimmune Syndrome (PAS)

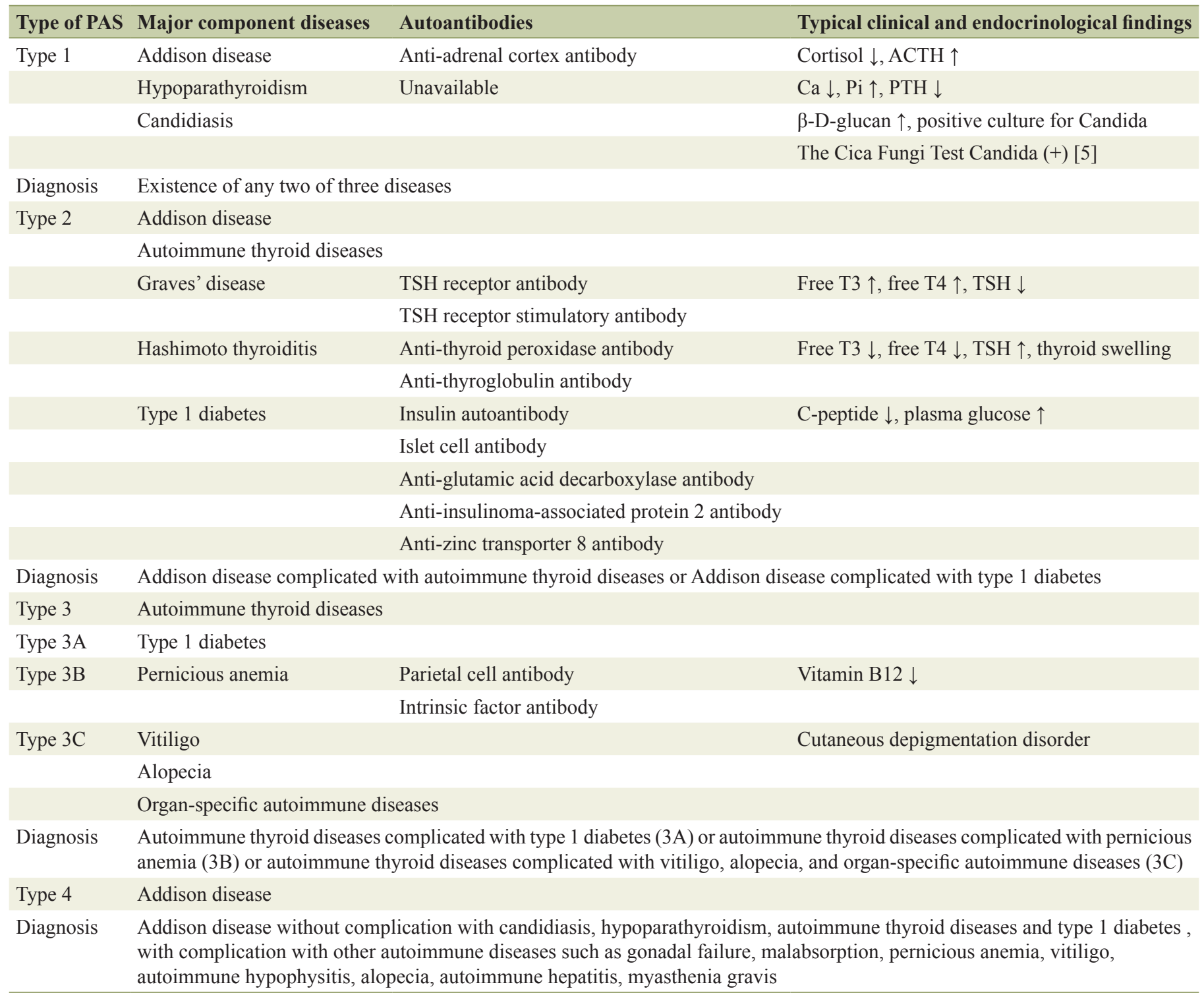

ACTH: adrenocorticotropic hormone; Ca: calcium; Pi: inorganic phosphorus; PTH: parathyroid hormone; T3: triiodothyronine; T4: thyroxine; TSH: thyroid-stimulating hormone. 